\title{
How should liver hypertrophy be stimulated? A comparison of upfront associating liver partition and portal vein ligation for staged hepatectomy (ALPPS) and portal vein embolization (PVE) with rescue possibility
}

\author{
Ernesto Sparrelid ${ }^{1}$, Kristina Hasselgren ${ }^{2}$, Bård Ingvald Røsok ${ }^{3}$, Peter Nørgaard Larsen ${ }^{4}$, Nicolai Aagaard \\ Schultz $^{4}$, Ulrik Carling ${ }^{5}$, Eva Fallentin ${ }^{6}$, Stefan Gilg ${ }^{1}$, Per Sandström ${ }^{2}$, Gert Lindell ${ }^{7}$, Bergthor Björnsson ${ }^{2}$ \\ ${ }^{1}$ Department of Clinical Science, Intervention and Technology, Division of Surgery, Karolinska Institute, Karolinska University Hospital, Stockholm, \\ Sweden; ${ }^{2}$ Department of Surgery and Clinical and Experimental Medicine, Linköping University, Linköping, Sweden; ${ }^{3}$ Department of Hepato- \\ Pancreato-Biliary Surgery, Oslo University Hospital, Oslo, Norway; ${ }^{4}$ Department of Surgical Gastroenterology and Transplantation, Rigshospitalet, \\ University of Copenhagen, Denmark; ${ }^{5}$ Department of Radiology and Nuclear Medicin, Rikshospitalet, Oslo University Hospital, Oslo, Norway; \\ ${ }^{6}$ Department of Radiology, Rigshospitalet, University of Copenhagen, Copenhagen, Denmark; ${ }^{7}$ Department of Surgery, Skane University Hospital, \\ Lund, Sweden \\ Contributions: (I) Conception and design: E Sparrelid, P Sandström, G Lindell, B Björnsson; (II) Administrative support: None; (III) Provision \\ of study materials or patients: All authors; (IV) Collection and assembly of data: All authors; (V) Data analysis and interpretation: E Sparrelid, P \\ Sandström, G Lindell, B Björnsson; (VI) Manuscript writing: All authors; (VII) Final approval of manuscript: All authors. \\ Correspondence to: Bergthor Björnsson. Department of Surgery and Clinical and Experimental Medicine, Linköping University, Linköping, Sweden. \\ Email: bergthor.bjornsson@liu.se.
}

Background: The role of associating liver partition and portal vein ligation for staged hepatectomy (ALPPS) in comparison to portal vein embolization (PVE) is debated. The aim of this study was to compare successful resection rates (RR) with upfront ALPPS vs. PVE with rescue ALPPS on demand and to compare the hypertrophy of the liver between ALPPS and PVE plus subsequent rescue ALPPS.

Methods: A retrospective analysis of all patients treated with PVE for colorectal liver metastasis (CRLM) or ALPPS (any diagnosis, rescue ALPPS included) at five Scandinavian university hospitals during the years 2013-2016 was conducted. A Chi-square test and a Mann-Whitney U test were used to assess the difference between the groups. A successful RR was defined as liver resection without a 90-day mortality.

Results: A total of 189 patients were included. Successful RR was in $84.5 \%$ of the patients with ALPPS upfront and in $73.3 \%$ of the patients with PVE and rescue ALPPS on demand $(\mathrm{P}=0.080)$. The hypertrophy of the future liver remnants (FLRs) with ALPPS upfront was 71\% (48-97\%) compared to 96\% (82-113\%) after PVE and rescue ALPPS (P=0.010).

Conclusions: Upfront ALPPS offers a somewhat higher successful RR than PVE with rescue ALPPS on demand. The sequential combination of PVE and ALPPS leads to a higher overall degree of hypertrophy than upfront ALPPS.

Keywords: Associating liver partition and portal vein ligation for staged hepatectomy (ALPPS); liver surgery; colorectal liver metastases (CRLM); portal vein embolization (PVE)

Submitted Aug 04, 2019. Accepted for publication Oct 08, 2019.

doi: $10.21037 /$ hbsn.2019.10.36

View this article at: http://dx.doi.org/10.21037/hbsn.2019.10.36 


\section{Introduction}

Currently, one of the main reasons liver malignancies are considered to be non-resectable is that the future liver remnants (FLRs) are too small (1). Until recently, the available methods for increasing the size of an FLR were portal vein embolization (PVE) and portal vein ligation, which are collectively known as portal vein occlusion (PVO). In addition, a two stage hepatectomy (TSH) without PVO increases the size of the FLR to some extent but is rarely used currently $(2,3)$. In 2012, a new method, associating liver partition and portal vein ligation for staged hepatectomy (ALPPS), was published after having been briefly reported in previous studies $(4,5)$. The main difference between ALPPS and the previous methods was the degree of hypertrophy of the FLR, the resection rate and the frequencies of complications and mortalities. Since the introduction of ALPPS, studies have demonstrated that it is more appropriate for use in the settings of colorectal liver metastasis (CRLM) than in primary liver and biliary malignancies (6). Furthermore, it has been demonstrated in a randomized trial that the use of ALPPS in patients with CRLM indeed increased the resection rate compared to the use of TSH with PVO, without increases in complications or mortalities (7). Few studies have reported on the use of ALPPS as a rescue treatment when PVE fails to induce sufficient hypertrophy of the FLR, and the total hypertrophy after the uses of both PVE and the rescue ALPPS has not been reported $(8,9)$. The increased morbidity that is initially observed with the use of ALPPS, compared to conventional methods that increase FLR volume, does not seem to apply to CRLM. Nevertheless, the ability to resect liver metastases with the use of only one procedure could be beneficial. Therefore, the main aims of this study were to compare the successful resection rates after the ALPPS upfront with PVE and ALPPS on demand as rescue procedure and to compare the hypertrophy of the FLR after the use of ALPPS upfront to the hypertrophy that is achieved with the use of sequential PVE and rescue ALPPS. We present the following article in accordance with the STROBE reporting checklist (available at http://dx.doi. org/10.21037/hbsn.2019.10.36).

\section{Methods}

Consecutive patients from five Scandinavian hepatobiliary centres who were treated with either PVE for CRLM or ALPPS (upfront or rescue ALPPS) between 2013 and 2016 were included in a retrospective analysis, which was approved by the ethical committees or by the data protection officials at the respective centres. Due to the expected paucity of the rescue ALPPS, all diagnoses were included in the comparison of hypertrophy with ALPPS upfront and PVE plus rescue ALPPS, in order to increase the statistical power. A portion of the data was prospectively collected within the Scandinavian LIGRO trial, and some of the patients had been previously reported on in various studies (7-16). Data were collected on demographics, diagnoses, oncological treatments, methods for inducing FLR hypertrophy, volumetries of the livers, perioperative variables and postoperative courses. The methods of volumetry were not standardized among the hospitals, but the same method was applied in each patient at different occasions. The methods and materials of PVE were also not standardized, but were applied according to the clinical routines at each of the participating centres. The methods of ALPPS/liver resection and PVE at different participating centres have been previously described (7). The primary outcome (successful resection rate) was defined as a completed liver resection without 90-day mortality, and the secondary outcome variable (FLR hypertrophy) was defined as a volume increase of the FLR from before the first attempt to stimulate hypertrophy until the last measurement before the liver resection. Complication rates and mortalities were calculated up to 90 days after the final intervention and according to the intention-to-treat principle. The Clavien-Dindo classification system was used to assess complications (17). The decision to apply rescue ALPPS was not standardized between the participating centres but was based on a multidisciplinary board assessment at each hospital. Similarly, the time between PVE and the radiological assessment was not standardized but was performed according to clinical practice. Estimated total liver volumes (eTLVs) were calculated according to a previously described method (18). The degree of hypertrophy was calculated as the sFLR before resectionthe sFLR before the intervention to induce hypertrophy. The kinetic growth rate (KGR) was calculated as the degree of hypertrophy divided by the time (in weeks) between the measurements and is presented in \%/week (19).

Patients undergoing PVE were subdivided into four groups depending on sFLR before intervention in order to access the proportion of successful resection after only PVE.

The study was conducted in accordance with the Declaration of Helsinki (as revised in 2013). The study was approved by ethics board in all participating countries (Dnr 2015/107-31, Dnr: 2019-01297, Dnr: 098-14, H-4-2014- 


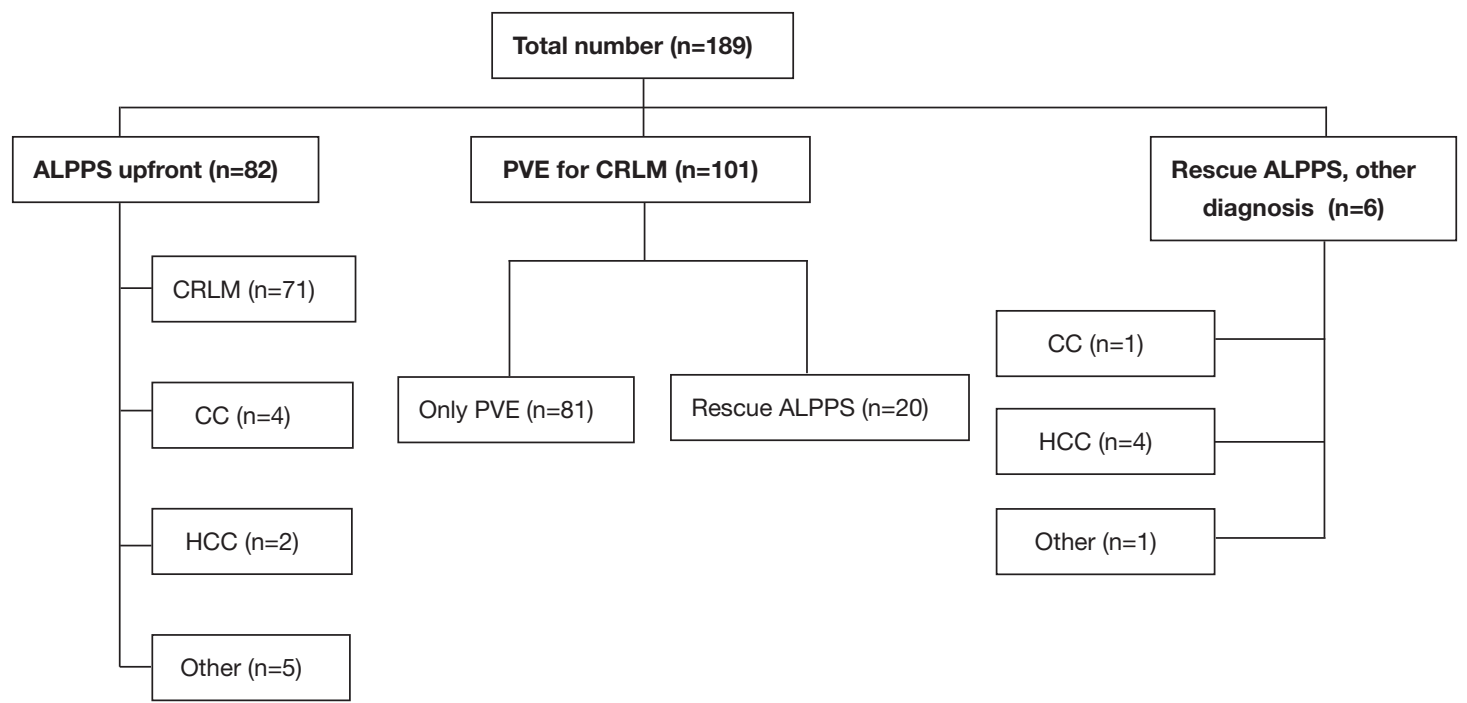

Figure 1 Flow chart of the included patients. CRLM, colorectal liver metastases; CC, cholangiocarcinoma; HCC, hepatocellular carcinoma; PVE, portal vein embolization; ALPPS, associating liver partition and portal vein ligation for staged hepatectomy.

Table 1 The distribution of 189 included patients between the participating centers

\begin{tabular}{lccc}
\hline Study center & PVE & ALPPS & Rescue ALPPS \\
\hline Oslo & 56 & 23 & 10 \\
Stockholm & 19 & 24 & 9 \\
Linköping & 7 & 15 & 6 \\
Copenhagen & 8 & 13 & 0 \\
Lund & 11 & 7 & 1 \\
\hline
\end{tabular}

PVE, portal vein embolization; ALPPS, associating liver partition and portal vein ligation for staged hepatectomy.

034, 2014/425 and 2014/428), and informed consent was deemed unnecessary by the ethics boards.

\section{Statistical analysis}

Statistical analyses were performed with IBM SPSS Statistics 25. The continuous data were compared with Mann-Whitney $U$ tests, and the categorical data were assessed with chi square tests. Data are presented as medians (interquartile ranges) or numbers (\%). A $\mathrm{P}$ value of $<0.05$ was considered to be statistically significant.

\section{Results}

A total of 189 patients were included in the study, with
172 patients (with CRLM) included in the analysis of the resection rates with ALPPS upfront compared to PVE and rescue ALPPS on demand. For the analysis of hypertrophy with ALPPS compared to PVE with subsequent rescue ALPPS, 108 patients (all diagnoses) were included (Figure 1). Table 1 shows the distribution of the included patients between participating centres. Most of the patients treated with ALPPS and rescue ALPPS have been included in previous analysis. The baseline characteristics of the patients with CRLM were similar between the groups, except for significantly larger tumours that were present in the ALPPS upfront group (Table 2). For the comparison of the baseline characteristics between ALPPS upfront (all diagnoses) and PVE plus rescue ALPPS, the only significant difference was a smaller sFLR in the PVE plus rescue ALPPS group at the beginning of treatment (Table 3).

\section{Subgroup analysis CRLM}

More patients in the ALPPS upfront group had successful resections than the patients in the PVE group although this difference was not statistically significant $(84.5 \%$ vs. $73.2 \%, \mathrm{P}=0.08)$. This result was not accompanied with any significant increase in post-operative 90 -day complications ( $31.0 \%$ vs. $24.8 \%, \mathrm{P}=0.37)$ or mortality $(11.3 \%$ vs. $8.9 \%$, $\mathrm{P}=0.61$ ) (Table 4). The reason for not achieving successful resections was, due to the post-operative mortalities in 8 of the ALPPS patients and in 3 it was due to intraoperative 
Table 2 Baseline characteristics for comparison of upfront ALPPS and PVE with rescue ALPPS on demand for CRLM

\begin{tabular}{lccc}
\hline Characteristic & ALPPS $(\mathrm{n}=71)$ & PVE $(\mathrm{n}=101)$ & $\mathrm{P}$ \\
\hline Age, years, median [IQR] & $65[56.8-69.3]$ & $66[59.4-72.5]$ & 0.24 \\
Gender, M/F & $48 / 23$ & $67 / 34$ & 0.86 \\
eTLV, mL, median [IQR] & $1,655[1,510-1,816]$ & $1,693[1,459-1,856]$ & 0.65 \\
SFLR, \%, median [IQR] & $21.8[18.6-25.5]$ & $20.9[17.4-25.3]$ & 0.33 \\
Number of lesions, median [IQR] & $6[5-9]$ & $6[3-8]$ & 0.27 \\
Size of largest lesion, mm, median [IQR] & $44.0[28.5-66.5]$ & $34[20-60]$ & 0.021 \\
Chemotherapy, number (\%) & $69(97.2)$ & $96(95.0)$ & 0.49 \\
\hline
\end{tabular}

ALPPS, associating liver partition and portal vein ligation for staged hepatectomy; PVE, portal vein embolization; CRLM, colorectal liver metastasis.

Table 3 Baseline characteristics for comparison of upfront ALPPS and sequential PVE plus rescue ALPPS

\begin{tabular}{lccc}
\hline Characteristic & ALPPS $(\mathrm{n}=82)$ & PVE/ALPPS $(\mathrm{n}=26)$ & $\mathrm{P}$ \\
\hline Age, years, median [IQR] & $65[56.3-70.0]$ & $67.5[61.4-71.8]$ & $19 / 7$ \\
Gender, M/F & $52 / 30$ & $1,727[1,618-1,870]$ & 0.23 \\
eTLV, mLSS, median [IQR] & $1,630[1,476-1,818]$ & $17.5[14.6-20.9]$ & 0.10 \\
sFLR, \%, median [IQR] & $21.6[17.7-25.4]$ & $6[1-8.8]$ & 0.004 \\
Number of lesions, median [IQR] & $6[4-9]$ & $60[30-95]$ & 0.50 \\
Size of largest lesion, mm, median [IQR] & $45[30-70]$ & $21(80.8)$ & 0.29 \\
Chemotherapy, number (\%) & $72(87.8)$ & 0.37 & \\
\hline
\end{tabular}

ALPPS, associating liver partition and portal vein ligation for staged hepatectomy; PVE, portal vein embolization.

Table 4 Outcomes in 172 patients with CRLM, treated with ALPPS upfront or PVE with rescue ALPPS on demand

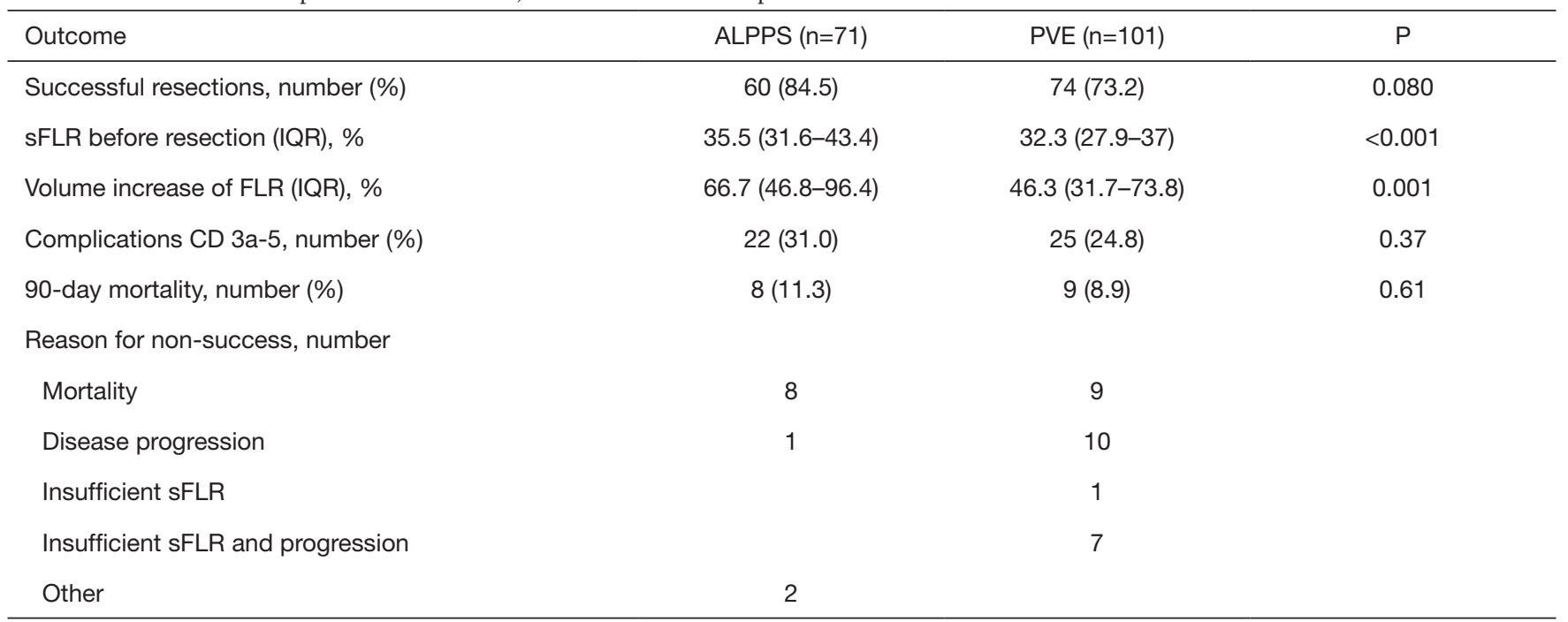

CRLM, colorectal liver metastasis; ALPPS, associating liver partition and portal vein ligation for staged hepatectomy; PVE, portal vein embolization; sFLR, standardized future liver remnant; IQR, interquartile range; CD, Clavien-Dindo. 
Table 5 Successful resection rates for PVE depending on different sFLRs before PVE

\begin{tabular}{lcccc}
\hline \multirow{2}{*}{ Outcome } & \multicolumn{4}{c}{ sFLR } \\
\cline { 2 - 5 } & $<15 \%$ & $15-20 \%$ & $20.1-25 \%$ & $>25 \%$ \\
\hline Unsuccessful & $4(23.5)$ & $7(23.3)$ & $8(29.6)$ & $8(29.6)$ \\
Rescue ALPPS & $7(41.2)$ & $8(26.7)$ & $3(11.1)$ & $0(0)$ \\
PVE only & $6(35.3)$ & $15(50.0)$ & $16(59.3)$ & $19(70.4)$ \\
\hline
\end{tabular}

sFLR, standardized future liver remnant; PVE, portal vein embolization; ALPPS, associating liver partition and portal vein ligation for staged hepatectomy.

Table 6 Volume increases of the FLR after ALPPS upfront compared to rescue ALPPS

\begin{tabular}{lccc}
\hline Volume & ALPPS $(\mathrm{n}=82)$ & Rescue ALPPS $(\mathrm{n}=26)$ & $\mathrm{P}$ \\
\hline sFLR before resection, (IQR), \% & $35.2(31.5-43.2)$ & $34(30.3-40.2)$ & 0.46 \\
sFLR after PVE, (IQR), \% & NA & $21.8(18.6-26.2)$ & NA \\
Volume increase of FLR, (IQR), \% & $70.9(47.3-96.7)$ & $96(82.4-113.1)$ & 0.010 \\
Volume increase after PVE, (IQR), \% & NA & $27.9(16.6-38.4)$ & NA \\
Volume increase of ALPPS & $70.9(47.3-96.7)$ & $52(38.4-75.4)$ & 0.045 \\
\hline
\end{tabular}

FLRs, future liver remnants; IQR, interquartile range; ALPPS, associating liver partition and portal vein ligation for staged hepatectomy; PVE, portal vein embolization.

findings (sever cirrhosis and injury to the left portal vein at stage 1 as well as non-resectable disease at stage 2 in one patient). Three of the patients that died within 90 days had insufficient growth (sFLR $<30 \%$ ) but high KGRs (4.5-11\%/week) motivating resection. The volume increase that was observed among the successfully resected patients after PVE was only $42.3 \%$ (31.3-57.6\%). In the PVE group, the reasons for not achieving successful resections were tumour progression, mortality and insufficient growth of the FLR. Two of the patients who died within 90 days had sFLR $<30 \%$ at the time of resection, and the KGR was $<2 \% /$ week $(1.1 \%$ and $1.3 \%$ for the two patients).

When the patients in the PVE group were divided into four groups (according to the sFLR sizes before PVE (Table 5), there were significantly more successful resections without the use of rescue ALPPS in those with sFLR > 25\% than in those with sFLR $<15 \%(\mathrm{P}=0.022)$, whereas the overall successful resection rate did not differ between the groups.

The lowest pre-PVE sFLR resulting in sFLR $>30 \%$ without rescue ALPPS was $17.4 \%$, whereas the lowest prePVE sFLR resulting in a successful resection without rescue ALPPS was $12.7 \%$. The KGR of the 21 patients with prePVE sFLR $<20 \%$ who underwent successful resections without rescue ALPPS was 2.1\%/week (1.6-2.9\%/week), and only 5 patients (24\%) had sFLR >30\% before resection.

\section{ALPPS upfront vs. PVE followed by rescue ALPPS}

The volume increase of the FLR in the rescue ALPPS group from pre-PVE until the time of resection was $96 \%$ (82-113\%), compared to $71 \%$ in the ALPPS group $(48-97 \%)(\mathrm{P}=0.010)$. When only patients with CRLM were included, the corresponding growths were $95 \%$ $(86-114 \%)$ and $67 \%(47-96 \%)$ for the volume increase in the rescue ALPPS group and for the volume increase in the ALPPS group, respectively $(\mathrm{P}=0.004)$. Most of the volume increase in the rescue ALPPS group (all diagnoses) was observed after the addition of ALPPS, although this was less pronounced than in the ALPPS upfront group $(\mathrm{P}=0.045)$ (Table 6). No significant differences were observed between the patients with CRLM and the other diagnoses in the other groups (data not shown). The rates of severe complications ( $\geq 3 \mathrm{a}$, according to the ClavienDindo classification) was $28 \%$ in the ALPPS upfront group and $42 \%$ in the PVE/rescue ALPPS group $(\mathrm{P}=0.17)$. The 90 -day mortality was $9.8 \%$ and $11.5 \%$ respectively $(\mathrm{P}=0.79)$.

\section{Discussion}

Since the introduction of ALPPS, it has become 
increasingly clear that this method offers a higher possibility of liver resection when the FLR is insufficient compared to previously described methods. Despite the results from a recent randomized trial that compared ALPPS to TSH in patients with CRLM showing no differences in complications or mortalities, the ALPPS method is still debated; in addition, it demands the use of two surgical procedures (7). The current study demonstrates that a somewhat higher proportion of patients who were treated with ALPPS upfront for CRLM are successfully resected, compared to when PVE is applied and the ALPPS technique is used as a rescue method for inadequate volume increases. This study defined successful resections in an intentionto-treat manner by combining the resection rate and mortality in a similar way as previous study with resection rate as primary outcome and mortality as a secondary outcome (20). The reason for this definition is the previously reported high resection rate and increased mortality with ALPPS $(4,21)$. Therefore, it seems reasonable to combine these variables into a single endpoint. The results differed from the previously claimed high resection rates that were obtained with the ALPPS method mainly because the 90-day mortality rate was considered to be a failure in this study. At the same time, the resection rate after PVE is high, which is partially due to the addition of rescue ALPPS, when necessary (19). Although this study only included patients from hepatobiliary centres who received the ALPPS method, and the study period started after the introduction of ALPPS, it is possible that more patients in the PVE group could have been offered rescue ALPPS. In 8 (37\%) of the cases that failed in the PVE group, an insufficient volume increase was evident. Despite tumour progression being present in 7 of these cases, it is possible that the early addition of ALPPS could have changed this outcome. It has recently been demonstrated that the main volume increase after PVE occurs early; therefore, an earlier application of rescue ALPPS seems to be a reasonable strategy, when indicated (22).

The volume increase with PVE alone that was observed in this study is comparable to previous studies. Given that the goal of the treatment is a sFLR of $30 \%$, this goal would not have been obtained for any patient with sFLR $<17.4 \%$ at the beginning of the treatment, and only $10.6 \%$ of the patients with an initial sFLR of $<20 \%$ with PVE only would have obtained this goal. Although the physiology of liver hypertrophy is certainly more complex than our results describe, and the role of KGR in predicting postoperative failure should be acknowledged, the results indicate a low chance of successful resection when the sFLR is $<20 \%$ before PVE.

Earlier studies on rescue ALPPS have focused on comparing the volume increases that are gained with the ALPPS method in different settings $(8,9)$. This study investigated the total volume increase in rescue ALPPS and, despite the somewhat lower increase between the stages of ALPPS, the total volume increase of the FLR was significantly greater than with the use of ALPPS alone. This result further supports the idea of using sequential PVE with the first volume assessment within 1-2 weeks, followed by the use of rescue ALPPS, when necessary (22).

Tumour progression after PVE, making resection impossible can be interpreted as a selection tool to spare patients the suffering of surgery when the prognosis is particularly negative. On the other hand, it may as well be considered a failure of treatment as done in the current study. This comes from the fact that patients in Scandinavia are treated with chemotherapy before liver resections in the settings of advanced CRLM and at least stable disease has to be present to proceed to major liver resection. Thus the "test of time" may be less relevant than in cohorts that have not received chemotherapy. This study has not investigated the ultimate outcome of cancer surgery, survival. However, a small subset of the patients included in the current study have been analysed regarding early recurrence without signs of increased recurrence after ALPPS (23).

Despite the large number of included patients, this retrospective study had several limitations.

First, the methods that were used for PVE differed between the centres. Although strong evidence for the superiority of any embolization material is lacking, this heterogenicity may have influenced the findings. Furthermore, the retrospective nature of this study accounts for heterogenicity between patients regarding the time between PVO and radiological evaluation. Additionally, the study period started after the first publication concerning ALPPS, and the use of the method could be unevenly distributed. Thus, some of the patients in the PVE group were not evaluated for this new treatment possibility. However, half of the rescue ALPPS procedures were performed in the first half of the study period, and the other half of the procedures were performed in the second half of the study period. During the study period, the clinical routine procedure of assessing volume increases approximately 4 weeks after PVE was the standard method; therefore, it is possible that some patients experienced tumour progression that could have been avoided with an 
early application of rescue ALPPS. Conversely, the lack of liver function measurements in both this study and a previous study that showed early growth of the FLR at one week after PVE should result in caution concerning the use of volume as the sole measurement of resectability (22).

The comparison of rescue ALPPS to ALPPS upfront included patients with several different diagnoses, including both primary hepatobiliary malignancies and CRLM. Some of the patients received neoadjuvant chemotherapy, while other patients did not receive this therapy. Both the type of disease and the preoperative treatment may influence the ability of the liver to undergo hypertrophy; therefore, this heterogeneity may be seen as being limiting. On the other hand, both groups included these different patients and, in a subgroup analysis of only the patients with CRLM, the difference was essentially the same as for the entire cohort.

Finally, sequential PVE and hepatic vein embolization had not been used in any of the included patients. The role of this new and less invasive method remains to be evaluated.

\section{Conclusions}

ALPPS upfront offers a non-significant higher degree of successful resections than PVE with rescue ALPPS on demand. In addition, patients who are treated with sequential PVE and rescue ALPPS have a better chance of obtaining adequate FLRs than those who are treated with ALPPS upfront. Therefore, the initial application of PVE and the subsequent application of ALPPS seems to be reasonable and is currently the preferred treatment plan at the participating centres. Due to the tendency towards higher resection rate with ALPPS upfront in the era of long waiting time after PVE and recent results indicating non-linear hypertrophy after PVE, we suggest short waiting time after PVE in order to be able to apply other measures to stimulate hypertrophy when needed. The mortality found in the current study is of concern although it is similar to findings from the largest TSH experience published where the authors also suggest that earlier studies may have underestimated mortality (24). This calls for both careful discussions with the affected patients about risks and benefits of the treatment and pursue of less invasive alternatives to stimulate liver hypertrophy.

\section{Acknowledgments}

Funding: None.

\section{Footnote}

Reporting Checklist: The authors have completed the STROBE reporting checklist. Available at http://dx.doi. org/10.21037/hbsn.2019.10.36

Data Sharing Statement: Available at http://dx.doi. org/10.21037/hbsn.2019.10.36

Conflicts of Interest: All authors have completed the ICMJE uniform disclosure from (available at http://dx.doi. org/10.21037/hbsn.2019.10.36). The authors have no conflicts of interest to declare.

Ethical Statement: The authors are accountable for all aspects of the work in ensuring that questions related to the accuracy or integrity of any part of the work are appropriately investigated and resolved. The study was conducted in accordance with the Declaration of Helsinki (as revised in 2013). The study was approved by ethics board in all participating countries (Dnr 2015/107-31, Dnr: 2019-01297, Dnr: 098-14, H-4-2014-034, 2014/425 and 2014/428), and informed consent was deemed unnecessary by the ethics boards.

Open Access Statement: This is an Open Access article distributed in accordance with the Creative Commons Attribution-NonCommercial-NoDerivs 4.0 International License (CC BY-NC-ND 4.0), which permits the noncommercial replication and distribution of the article with the strict proviso that no changes or edits are made and the original work is properly cited (including links to both the formal publication through the relevant DOI and the license). See: https://creativecommons.org/licenses/by-nc-nd/4.0/.

\section{References}

1. Charnsangavej C, Clary B, Fong Y, et al. Selection of patients for resection of hepatic colorectal metastases: expert consensus statement. Ann Surg Oncol 2006;13:1261-8.

2. Albati NA, Korairi AA, Hasan IA, et al. Outcomes of staged hepatectomies for liver malignancy. World J Hepatol 2019;11:513-21.

3. Lam VW, Laurence JM, Johnston E, et al. A systematic review of two-stage hepatectomy in patients with initially unresectable colorectal liver metastases. HPB (Oxford) 2013;15:483-91.

4. Schnitzbauer AA, Lang SA, Goessmann H, et al. Right portal vein ligation combined with in situ splitting induces 
rapid left lateral liver lobe hypertrophy enabling 2-staged extended right hepatic resection in small-for-size settings. Ann Surg 2012;25 5:405-14.

5. Baumgart J, Lang S, Lang H. A new method for induction of liver hypertrophy prior to right trisectionectomy: a report of three cases. HPB 2011;13:71-2.

6. Schadde E, Ardiles V, Robles-Campos R, et al. Early survival and safety of ALPPS: first report of the International ALPPS Registry. Ann Surg 2014;260:829-36; discussion 36-8.

7. Sandström P, Rosok BI, Sparrelid E, et al. ALPPS Improves Resectability Compared With Conventional Two-stage Hepatectomy in Patients With Advanced Colorectal Liver Metastasis: Results From a Scandinavian Multicenter Randomized Controlled Trial (LIGRO Trial). Ann Surg 2018;267:833-40.

8. Enne M, Schadde E, Bjornsson B, et al. ALPPS as a salvage procedure after insufficient future liver remnant hypertrophy following portal vein occlusion. HPB (Oxford) 2017;19:1126-9.

9. Sparrelid E, Gilg S, Brismar TB, et al. Rescue ALPPS is efficient and safe after failed portal vein occlusion in patients with colorectal liver metastases. Langenbecks Arch Surg 2017;402:69-75.

10. Linecker M, Kambakamba P, Reiner CS, et al. How much liver needs to be transected in ALPPS? A translational study investigating the concept of less invasiveness. Surgery 2017;161:453-64.

11. Linecker M, Bjornsson B, Stavrou GA, et al. Risk Adjustment in ALPPS Is Associated With a Dramatic Decrease in Early Mortality and Morbidity. Ann Surg 2017;266:779-86.

12. Røsok BI, Bjornsson B, Sparrelid E, et al. Scandinavian multicenter study on the safety and feasibility of the associating liver partition and portal vein ligation for staged hepatectomy procedure. Surgery 2016;159:1279-86.

13. D'Haese JG, Neumann J, Weniger M, et al. Should ALPPS be Used for Liver Resection in Intermediate-Stage HCC? Ann Surg Oncol 2016;23:1335-43.

14. Björnsson B, Sparrelid E, Rosok B, et al. Associating liver partition and portal vein ligation for staged hepatectomy in patients with colorectal liver metastases--Intermediate oncological results. Eur J Surg Oncol 2016;42:531-7.

15. Björnsson B, Sparrelid E, Hasselgren K, et al. Associating Liver Partition and Portal Vein Ligation for Primary Hepatobiliary Malignancies and Non-Colorectal Liver Metastases. Scand J Surg 2016;105:158-62.

16. Björnsson B, Gasslander T, Sandström P. In situ split of the liver when portal venous embolization fails to induce hypertrophy - a report of two cases. Case Rep Surg 2013;2013:238675.

17. Dindo D, Demartines N, Clavien PA. Classification of surgical complications: a new proposal with evaluation in a cohort of 6336 patients and results of a survey. Ann Surg 2004;240:205-13.

18. Vauthey JN, Abdalla EK, Doherty DA, et al. Body surface area and body weight predict total liver volume in Western adults. Liver Transpl 2002;8:233-40.

19. Shindoh J, Truty MJ, Aloia TA, et al. Kinetic growth rate after portal vein embolization predicts posthepatectomy outcomes: toward zero liver-related mortality in patients with colorectal liver metastases and small future liver remnant. J Am Coll Surg 2013;216:201-9.

20. Schadde E, Ardiles V, Slankamenac K, et al. ALPPS offers a better chance of complete resection in patients with primarily unresectable liver tumors compared with conventional-staged hepatectomies: results of a multicenter analysis. World J Surg 2014;38:1510-9.

21. Nadalin S, Capobianco I, Li J, et al. Indications and limits for associating liver partition and portal vein ligation for staged hepatectomy (ALPPS). Lessons Learned from 15 cases at a single centre. Z Gastroenterol 2014;52:35-42.

22. Hasselgren K, Sandstrom P, Rosok BI, et al. Future Liver Remnant (FLR) Increase in Patients with Colorectal Liver Metastases Is Highest the First Week After Portal Vein Occlusion : FLR Increase in Patients with CRLM Is Highest the First Week After PVO. J Gastrointest Surg 2019;23:556-62.

23. Røsok BI, Host-Brunsell T, Brudvik KW, et al. Characterization of early recurrences following liver resection by ALPPS and two stage hepatectomy in patients with colorectal liver-metastases and small future liver remnants; a translational substudy of the LIGRO-RCT. HPB (Oxford) 2019;21:1017-23.

24. Regimbeau JM, Cosse C, Kaiser G, et al. Feasibility, safety and efficacy of two-stage hepatectomy for bilobar liver metastases of colorectal cancer: a LiverMetSurvey analysis. HPB (Oxford) 2017;19:396-405.

Cite this article as: Sparrelid E, Hasselgren K, Røsok BI, Larsen PN, Schultz NA, Carling U, Fallentin E, Gilg S, Sandström P, Lindell G, Björnsson B. How should liver hypertrophy be stimulated? A comparison of upfront ALPPS and PVE with rescue possibility. HepatoBiliary Surg Nutr 2021;10(1):1-8. doi: 10.21037/hbsn.2019.10.36 